\title{
Evaluation of Charge-Transfer Rates in Fullerene-Based Donor- Acceptor Dyads with Different Density Functional Approximations
}

\author{
Pau Besalú-Sala, ${ }^{a}$ Alexander A. Voityuk *ab Josep M. Luis ${ }^{a}$ and Miquel Solà*a \\ The shift towards renewable energy is one of the main challenges of this generation. Dye-sensitized solar cells (DSSC), based \\ on donor-acceptor architectures, can help on this transition as they present excellent photovoltaic efficiencies yet cheap \\ and simple manufacturing. For molecular heterojunctions DSSCs, donor-acceptor pairs are linked in a covalent manner, \\ which facilitates their tailoring and rational design. Nevertheless, reliable computational characterization of charge transfer \\ rate constants $\left(k_{C T}\right)$ is needed to speed this development process up. In this context, the performance of time-dependent \\ density functional theory for the calculation of $k_{C T}$ 's in donor-acceptor fullerene-based dyads has not been benchmarked \\ yet. Herein, we present a detailed analysis on the performance of seven well-known density functional approximations \\ (DFAs) for this type of systems, focusing on several parameters as the reorganization energies $(\lambda)$, electronic couplings ( $\left.V_{D A}\right)$, \\ and Gibbs energies $\left(\Delta \mathrm{G}^{0} \mathrm{CT}\right)$, as well as in the final rate constants. The amount of exact exchange at short range (SR) and long \\ range (LR) electron-electron distances (and the transition from SR to LR) turned out to be key for the success of the \\ prediction. The tuning of these parameters improves significantly the performance of current DFAs.
}

\section{Introduction}

Renewable energy boosting is the $7^{\text {th }}$ target of the United Nation member states' 2030 Sustainable Agenda Development ${ }^{1}$ whose premise is to accelerate the transition to a competitive low-carbon economy. The production of non-contaminating renewable energy is undoubtedly one of the biggest challenges facing humanity nowadays. The Earth receives just a tiny fraction of the Sun's colossal power output. But even that tiny fraction represents 120000 trillion Watts and, consequently, we would need to harvest less than $0.02 \%$ to fulfil the energy consumed on the planet. ${ }^{2}$ Energy from the Sun can be collected with solar cells, which are devices that convert absorbed incident sunlight into electricity. Most current solar cells are silicon-based inorganic cells. These cells have a high cost, heavy weight, and their production requires high energy consumption and leads to environmental pollution. ${ }^{3}$ Thanks to their excellent photovoltaic efficiency and simple and low cost production process, dye-sensitized solar cells (DSSC) are a promising member of the so-called $3^{\text {rd }}$ generation of photovoltaic devices. Fullerenes and derivatives have emerged as promising materials for the design of efficient DSSCs. ${ }^{4-8}$ Two different types of DSSCs are usually distinguished: bulk heterojunctions ${ }^{9}$ (BHJs) where the donor and acceptor (D/A) moieties are not covalently connected, and molecular heterojunctions ${ }^{10}$ (mHJs) with covalently linked donor-acceptor (D-A) dyads. Main advantage of $\mathrm{mHJs}$ is that charge transfer (CT) processes can be rationally designed since a better structural control and charge mobility tuning can be achieved within this approach.

Tuning the electronic and structural parameters of solar cells for optimum performance is a difficult task because several tradeoffs should be considered. The experimental approach to optimize DSSCs is heuristic, mainly based on trial and error. In this sense, computational modelling of molecular interactions and charge transport in macromolecular photovoltaic materials is the most rational approach for the design of highperformance DSSCs. Computational modelling of the power conversion efficiency of DSSCs requires the knowledge of the charge transfer rate constants $\left(\mathrm{k}_{\mathrm{CT}}\right){ }^{11}$ In some cases, $\mathrm{k}_{\mathrm{CT}}$ are known experimentally, but in most cases, the most efficient way for designing or tuning DSSCS is to estimate the $k_{C T}$ from calculations, and select the most promising D-A dyads to be used as building blocks for mHJs DSSCs, which should also contain anchoring groups to interact with $\mathrm{TiO}_{2}$ electron acceptors. ${ }^{12-15}$

There a number of studies that have benchmarked different density functional approximations (DFAs) for the calculation of excited states based on time-dependent density functional theory (TDDFT). ${ }^{16-23}$ It is widely accepted that standard functionals underestimate the excitation energies of CT states and only range-separated DFAs provide good estimates of these states. ${ }^{24-29}$ To our knowledge, despite its relevance, no benchmarks of DFAs for the calculation of $\mathrm{k}_{\mathrm{CT}}$ in D-A(fullerene) dyads are available yet. The present work aims to fill this void. In particular, we compare six well-known range-separated DFAs (CAM-B3LYP, $\omega B$ 97xD, LC-whPBE, LC-TPSS, LC-BLYP, M11) and one meta-hybrid-generalized gradient approximation (MHGGA) functional, MN15. For these seven DFAs, we calculate $k_{C T}$ of four D-A dyads for which experimental charge transfer rate constants are available.

\section{Computational Methods}

The ground state geometry for each structure was optimized at the B3LYP-D3(BJ)/6-311G(d,p) level of theory, ${ }^{30-32}$ including dispersion interactions through D3 empirical model of Grimme, with the Becke-Johnson damping. ${ }^{33}$, 34 Previous studies have demonstrated that CT in DSSC are mostly purely electronic processes and, for this reason, it is usually not necessary to account for the structural changes of the D-A dyads induced by the charge-transfer processes, ${ }^{35-37}$ except for the calculation of the reorganization energy. Nevertheless, in order to confirm whether this assumption holds for the systems studied herein, we have computed the root-mean-square deviation (RMSD) of atomic positions of $\mathrm{D}^{*}$ with respect to $\mathrm{D}^{+}$and $\mathrm{A}^{*}$ with respect to $A^{-}$, i.e. the two molecular fragments involved in the $C T$ reaction. The highest RMSD obtained was $<0.1 \AA$, confirming that one can address the $C T$ reactions of this manuscript as purely electronic processes (see ESI for further details). In addition, D-A dyads 
considered in this work are relatively rigid, and, therefore, we do not expect large conformational effects.

The donor and acceptor geometries needed to estimate the internal reorganization energy (vide infra) and the geometrical changes upon CS were obtained at the same level of theory as the ground state equilibrium geometries.

Hundred lowest-lying singlet excited states for each D-A pair were computed using the TDDFT formalism with the double- $\zeta$ with polarization Def2SVP basis set ${ }^{38}$ and the seven DFAs. The ground and excited state calculations were performed with the Gaussian16 package. ${ }^{39}$

\section{Analysis of excited states}

A quantitative analysis of exciton delocalization and charge separation is carried out in terms of the transition density. 40,41 It is convenient to perform the analysis using the Löwdin orthogonalized basis. The matrix ${ }^{\lambda} \mathbf{C}$, which contains the orthogonalized molecular orbital (MO) coefficients in the Löwdin orthogonalized basis, is obtained from the matrix $\mathbf{C}$, formed by the $\mathrm{MO}$ coefficients in the original basis; ${ }^{\lambda} \mathbf{C}=\mathbf{S}^{1 / 2} \mathbf{C}$, where $\mathbf{S}$ is the atomic orbital overlap matrix. The transition density matrix $\mathbf{T}$ for an excited state $\Phi^{*}$ constructed as a superposition of singly excited configurations (where an occupied $\mathrm{MO} \psi_{\mathrm{j}}$ in the ground state is replaced a virtual $\mathrm{MO} \psi_{\mathrm{a}}$ ) is given by:

$$
\boldsymbol{T}_{\alpha \beta}=\sum_{j a} A_{j \rightarrow a}{ }^{\lambda} \boldsymbol{C}_{\alpha j}{ }^{\lambda} \boldsymbol{C}_{\beta a}
$$

where $A_{j \rightarrow a}$ is the expansion coefficient and $\alpha$ and $\beta$ are atomic orbitals. A key quantity $\Omega(D, A)$ is determined by:

$$
\Omega(D, A)=\frac{1}{2} \sum_{\alpha \in D, \beta \in A}\left(\boldsymbol{T}_{\alpha \beta}\right)^{2}
$$

The weights of local excitations on $D$ and $A$ are $\Omega(D, D)$ and $\Omega(A, A)$. The weight of electron transfer configurations $D \rightarrow A$ and $A \rightarrow D$ is represented by $\Omega(D, A)$ and $\Omega(A, D)$, respectively. The index $C S=\Omega(D, A)-\Omega(A, D)$ describes charge separation between $D$ and $A$; the $C T=\Omega(D, A)+\Omega(A, D)$ is the total weight of CT configurations in the excited state $\Phi^{*}$. Note that in the situation when charge transfer $(D \rightarrow A)$ is equal to the back transfer $(A \rightarrow D)$ there is no charge separation between the fragments, and $C S^{D \rightarrow A}$ is equal to zero. With this methodology, CT states (CTS) and local excited states (LES) can be easily distinguished and identified. In LES, the excitation is mostly localized on a single fragment (CS $<0.1 \mathrm{e})$, whereas in CTS electron density is transferred between $\mathrm{D}$ and $\mathrm{A}$ (CS $>0.9 \mathrm{e}$ ). Mixed states have significant contributions of LE and CT.

\section{Solvent effects}

The equilibrium solvation energy $\mathrm{E}_{\mathrm{S}}^{\mathrm{eq}}$ of a molecule (in the ground or excited state) in the medium with the dielectric constant $\varepsilon$ was estimated using a COSMO-like polarizable continuum model ${ }^{42,43}$ in the monopole approximation:

$$
\mathrm{E}_{\mathrm{S}}^{\mathrm{eq}}(\mathrm{Q}, \varepsilon)=-\frac{1}{2} \mathrm{f}(\varepsilon) \mathrm{Q}^{+} \mathrm{DQ},
$$

where the $\mathrm{f}(\varepsilon)$ is the dielectric scaling factor, $f(\varepsilon)=1-1 / \varepsilon$ Q is the vector of $\mathrm{n}$ atomic charges in the molecular system, $\mathbf{D}$ is the $\mathrm{n} \times \mathrm{n}$ symmetric matrix determined by the shape of the boundary surface between solute and solvent. $\mathbf{D}=\mathbf{B}^{+} \mathbf{A}^{-1} \mathbf{B}$, where the $\mathrm{m} \times \mathrm{m}$ matrix $\mathbf{A}$ describes electrostatic interaction between $m$ surface charges and the $m \times n$ B matrix describes the interaction of the surface charges with $\mathrm{n}$ atomic charges of the solute. ${ }^{42,43}$ The GEPOL93 scheme ${ }^{44}$ was used to construct the molecular boundary surface.

The charge on atom $X$ in the excited state $\Phi^{*}, \mathrm{q}_{\mathrm{X}}^{*}$, was calculated as:

$$
\begin{gathered}
\mathrm{q}_{\mathrm{X}}^{*}=\mathrm{q}_{\mathrm{X}}^{0}+\Delta_{\mathrm{X}}, \\
\Delta_{\mathrm{X}}=\frac{1}{2} \sum_{\mathrm{Y} \neq \mathrm{X}} \sum_{\alpha \in \mathrm{X}, \beta \in \mathrm{Y}}\left(\mathbf{T}_{\alpha \beta} \mathbf{T}_{\alpha \beta}-\mathbf{T}_{\beta \alpha} \mathbf{T}_{\beta \alpha}\right)
\end{gathered}
$$

where $\mathrm{q}_{\mathrm{X}}^{0}$ is the charge on atom $\mathrm{X}$ in the ground state and $\Delta_{\mathrm{X}}$ is its change due to excitation.

The non-equilibrium solvation energy for the molecule in the excited state $\Phi^{*}$ can be estimated as: ${ }^{45}$

$$
\mathrm{E}_{\mathrm{S}}^{\text {neq }}\left(\mathbf{Q}^{\mathbf{0}}, \Delta, \varepsilon, \mathrm{n}^{2}\right)=\mathrm{f}(\varepsilon) \Delta^{+} \mathbf{D} \mathbf{Q}^{\mathbf{0}}-\frac{1}{2} \mathrm{f}\left(\mathrm{n}^{2}\right) \Delta^{+} \mathbf{D} \Delta,
$$

In Eq. (5), $\mathrm{n}^{2}$ (the refraction index squared) is the optical dielectric constant of the medium and the vector $\Delta$ describes the change of atomic charges, see Eq. (4b); and $\mathbf{Q}^{\mathbf{0}}$ is the vector of atomic charges in the ground state. By definition, the external (solvent) reorganization energy is the difference of the nonequilibrium (Eq. 5) and equilibrium (Eq. 3) solvation energies of the excited state.

\section{CT rates}

Within the nonadiabatic electron transfer theory, the CT process $D A \rightarrow D^{+} A^{-}$can be described with the Marcus equation: 46,47

$$
k_{C T}=\frac{2 \pi}{\hbar} V_{D A}^{2} \frac{1}{\sqrt{4 \pi \lambda k_{B} T}} \exp \left(-\frac{\left(\lambda+\Delta G_{C T}^{0}\right)^{2}}{4 \lambda k_{B} T}\right)
$$

where $\lambda$ is the total reorganization energy, $\Delta \mathrm{G}^{0}{ }_{\mathrm{CT}}$ is the Gibbs energy difference between the final and the initial states in standard conditions, $V_{D A}^{2}$ is the square of the electronic coupling of the donor and acceptor, $\mathrm{k}_{\mathrm{B}}$ is the Boltzmann constant, $\hbar$ is the reduced Planck constant, and $\mathrm{T}$ is the temperature, which was set to $298 \mathrm{~K}$ for all systems. The Marcus equation is widely used to estimate the rate of nonadiabatic electron transfer.

\section{Reorganization energy, $\lambda$}

The total reorganization energy (Figure 1 , right) is usually decomposed into the internal and external contributions $\left(\lambda_{\text {int }}\right.$ and $\left.\lambda_{\text {ext }}\right) . \lambda_{\text {int }}$ is the average of the energy required to distort the nuclear configuration from the $D^{+}-A^{-}\left((D-A)^{*}\right)$ equilibrium geometry to the equilibrium geometry of the $(D-A)^{*}\left(D^{+}-A^{-}\right)$state without transferring an electron. And $\lambda_{\text {ext }}$ is the corresponding energy required to change the slow (reorientational) part of the solvent polarization between both equilibrium geometries. In this study, $\lambda_{\text {int }}$ was computed considering isolated donor and acceptor, which contribute separately to the internal reorganization energy:

$$
\lambda_{\text {int }}=\lambda_{D}+\lambda_{A}
$$


where $\lambda_{\mathrm{D}}$ and $\lambda_{\mathrm{A}}$ are the reorganization energy of the donor and acceptor. In turn, $\lambda_{D}$ was estimated as

$$
\begin{gathered}
\lambda_{D}=\frac{1}{2}\left(\lambda_{D}^{\prime}+\lambda_{D}^{\prime \prime}\right) \\
\lambda_{D}^{\prime}=E_{n}^{\prime}(D)-E_{n}(D) \\
\lambda_{D}^{\prime \prime}=E_{\text {ion }}^{\prime}(D)-E_{\text {ion }}(D)
\end{gathered}
$$

$E_{n}(D)$ and $E_{i o n}(D)$ are the energy of the neutral and ionic states of donor computed at their equilibrium geometry, $E_{n}{ }^{\prime}(D)$ is the energy of the neutral state computed at the equilibrium geometry of the ionic state $D^{+} . E_{\text {ion }}{ }^{\prime}(D)$ is the energy of $D^{+}$, estimated at the equilibrium geometry of $D$. Similarly, we calculated $\lambda_{A}$ using equilibrium geometries of $A$ and $A^{-}$.

There are two contributions to the solvent polarization corresponding to fast electronic relaxation and a much slower dipole reorientation. During a vertical electronic excitation, the solvent does not have enough time to fully relax, and the situation is described by nonequilibrium solvation ( $\left.E_{\text {noneq }}\right)$ where only electron polarization is allowed. The equilibrium solvation $\left(\mathrm{E}_{\mathrm{eq}}\right)$ assumes both electronic and dipole relaxation. Accordingly, the external reorganization energy, $\lambda_{\text {ext }}$, is the difference between these two quantities $\left(E_{\text {noneq }}-E_{\text {eq }}\right)$. This scheme to estimate $\lambda_{\text {ext }}$ is widely accepted and is an extension of the two-sphere Marcus model. ${ }^{48}$

\section{Electronic coupling and changes in Gibbs energy}

There are a number of studies focused on the development of theoretical frameworks for the evaluation of electronic coupling. ${ }^{49-51}$ In this manuscript we made use of the Fragment Charge Difference method ( $F C D$ ) to derive the coupling of LES and CTS calculated with TDDFT. ${ }^{52}$ Within the two-states model, the D-A couplings reads

$$
V_{D A}=\frac{\left(E_{i}-E_{j}\right)\left|\Delta q_{i j}\right|}{\sqrt{\left(\Delta q_{i}-\Delta q_{j}\right)^{2}+4\left(\Delta q_{i j}\right)^{2}}}
$$

where $\Delta \boldsymbol{q}_{\boldsymbol{i}}$ and $\boldsymbol{\Delta} \boldsymbol{q}_{\boldsymbol{j}}$ are the difference in the donor and acceptor charges in the adiabatic states $\Phi_{\mathrm{i}}$ and $\Phi_{\mathrm{j}}$, respectively, and $\boldsymbol{\Delta} \boldsymbol{q}_{\boldsymbol{i j}}$ is the charge difference computed from the $\Phi_{\mathrm{i}} \rightarrow \Phi_{\mathrm{j}}$, transition density matrix.

The Franck-Condon excitations in solution and photoinduced charge transfer reactions in most $\mathrm{C}_{60}$-based $\mathrm{D}$-A dyads are summarized in Figure 1, left. First, the photo-sensible system gets excited by light to one high-in-energy LES, which then relaxes to the lowest LES, LES ${ }_{1}$. In this step, the electron-hole pair (exciton) is generated in the fullerene cage (usually $\mathrm{C}_{60}$ ). The following step is the electron-hole pair separation, in which one electron is transferred from the donor to the acceptor. The $\Delta \mathrm{G}^{0}$ of this step corresponds to the $\mathrm{CT}$ process $\left(\Delta \mathrm{G}^{0} \mathrm{CT}\right)$. Finally, the charge recombination occurs, thus leading to the recovery of the ground state. Note that CTSs are usually stabilized by polar environments much stronger than LESs and thus the solvent effects should be accounted for.

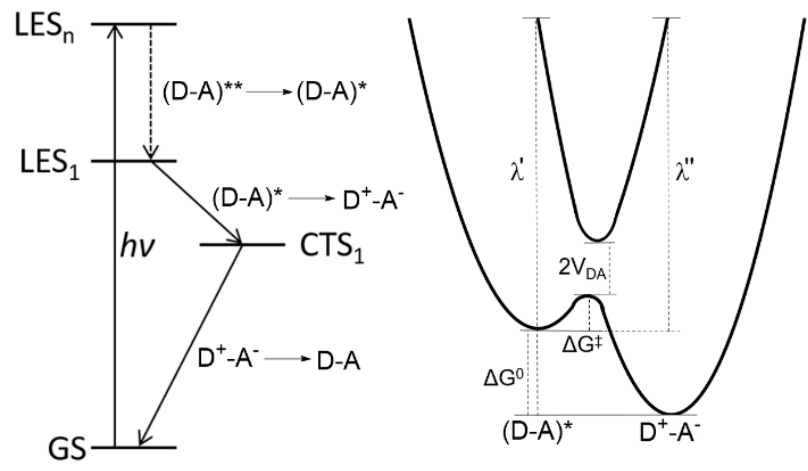

Figure 1. Left, the Franck-Condon excitation and photo-induced charge-transfer thermodynamic cycle in solution for donor-acceptor(fullerene) dyads. Right schematization of the reaction coordinate and the physical interpretation of $\lambda, V_{D A}$ and $\Delta \mathrm{G}^{0}$.

\section{Results and Discussion}

We have evaluated the performance of seven DFAs; namely MN15, ${ }^{53}$ M11, ${ }^{54}$ CAM-B3LYP, ${ }^{55} \omega B 97 \times D,{ }^{56}$ LC- $\omega h P B E^{57,} 58$ LCTPSS $^{59}$, and LC-BLYP ${ }^{30}, 31$ (the last six are range-separated functionals, and the last three are based on the range separation scheme from Hirao and coworkers ${ }^{60}$ ) for the calculation of charge transfer rates, $\mathrm{k}_{\mathrm{CT}}$, in $\mathrm{C}_{60}$-based donoracceptor pairs. This set of functionals were selected because they tend to be the best for CT studies among the huge variety of DFAs. ${ }^{24-27}$

The four molecular dyads studied (Figure 2 ) are $\mathbf{C}_{60}$-azulene $\left(\mathbf{C}_{60^{-}}\right.$ Az); ${ }^{61} \mathrm{C}_{59} \mathrm{~N}$-phtalocyanine $\left(\mathrm{C}_{59} \mathrm{~N}-\mathbf{P c}\right) ;{ }^{62}$ trans-2 $\mathbf{C}_{60}-\mathrm{ZnTPP}{ }^{63}$ and $\mathrm{C}_{60}$-triphenylamine $\left(\mathbf{C}_{60}\right.$-TPA). ${ }^{64}$ All of them were previously reported in the literature as functional materials with experimentally measured rate constants. In addition, these systems are relatively rigid and, consequently, conformational fluctuations play here a less important role than in other more flexible dyads. For these systems, we analysed the key parameters that affect $k_{\mathrm{CT}}$, thus $\lambda, \mathrm{V}_{\mathrm{DA}}$ and $\Delta \mathrm{G}^{0}{ }_{\mathrm{CT}}$.

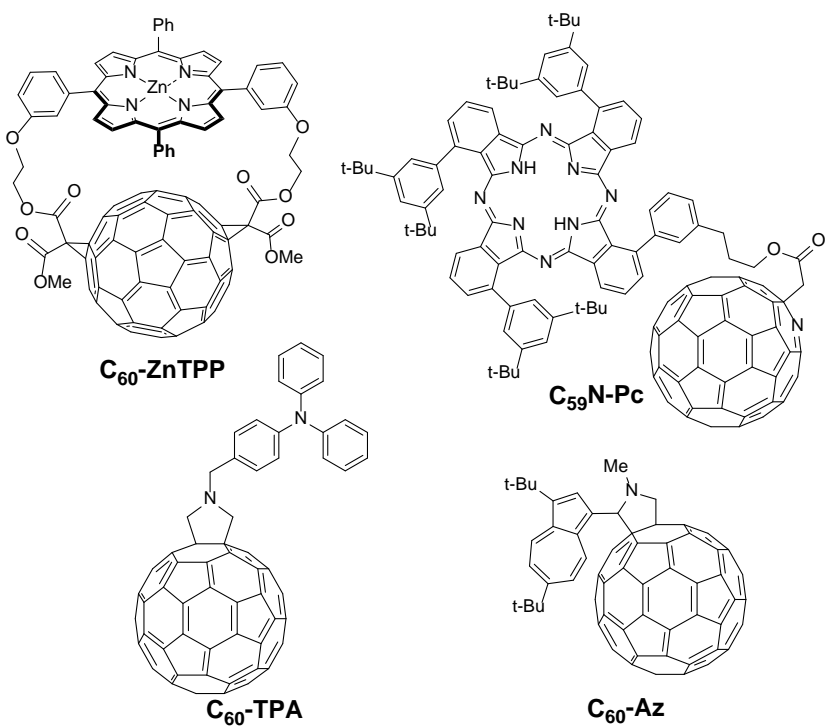

Figure 2. Donor-Acceptor dyads studied in this work. 


\section{Reorganization energy, $\lambda$}

The internal and external contributions to the reorganization energy, $\lambda_{\text {int }}$ and $\lambda_{\text {ext }}$, computed for all DFAs are listed in Table 1 (and Figures SI4-6 on the ESI). For each system, the mean and the standard deviation (SD) of both parameters for the DFAs studied were calculated. As seen, all DFAs give a similar value of $\lambda_{\text {int }}$ (SD does not exceed $0.05 \mathrm{eV}$ ). Therefore, the small variations in $\lambda_{\text {int }}$ cannot lead to a substantial change in the predicted $\mathrm{k}_{\mathrm{CT}}$. Higher variations are found for $\lambda_{\text {ext }}$, with largest SD of $0.16 \mathrm{eV}$.

Table 1. Internal and external reorganization energies. All values in eV.

\begin{tabular}{|c|c|c|c|c|}
\hline$\lambda_{\text {int }}(\mathrm{eV})$ & $\mathrm{C}_{60}-\mathrm{Az}$ & $\mathrm{C}_{60}-\mathrm{ZnTTP}$ & $\mathrm{C}_{59} \mathrm{~N}-\mathrm{Pc}$ & $\mathrm{C}_{60}-\mathrm{TPA}$ \\
\hline CAM-B3LYP & 0.24 & 0.28 & 0.13 & 0.21 \\
\hline MN15 & 0.23 & 0.27 & 0.13 & 0.19 \\
\hline$\omega B 97 x D$ & 0.24 & 0.28 & 0.13 & 0.22 \\
\hline M11 & 0.25 & 0.29 & 0.14 & 0.21 \\
\hline LC- $\omega$ hPBE & 0.26 & 0.30 & 0.14 & 0.24 \\
\hline LC-TPSS & 0.27 & 0.26 & 0.15 & 0.32 \\
\hline LC-BLYP & 0.27 & 0.31 & 0.15 & 0.26 \\
\hline Mean & 0.25 & 0.28 & 0.14 & 0.24 \\
\hline SD & 0.02 & 0.02 & 0.01 & 0.05 \\
\hline \multicolumn{5}{|l|}{$\lambda_{\text {ext }}(\mathrm{eV})$} \\
\hline CAM-B3LYP & 0.37 & 0.25 & 0.21 & 0.94 \\
\hline MN15 & 0.10 & 0.30 & 0.27 & 0.97 \\
\hline$\omega B 97 x D$ & 0.22 & 0.16 & 0.11 & 0.82 \\
\hline M11 & 0.21 & 0.22 & 0.21 & 0.82 \\
\hline LC- $\omega$ hPBE & 0.26 & 0.09 & 0.23 & 0.88 \\
\hline LC-TPSS & 0.24 & 0.06 & 0.24 & 0.89 \\
\hline LC-BLYP & 0.11 & 0.12 & 0.24 & 0.53 \\
\hline Mean & 0.21 & 0.17 & 0.22 & 0.84 \\
\hline SD & 0.09 & 0.09 & 0.05 & 0.15 \\
\hline
\end{tabular}

The relatively small values of $\lambda_{\text {int }}$ computed for the dyads are associated with a significant delocalization of excess charges generated on the donor and acceptor after CT. One may expect that in fullerene containing systems the changes in $\lambda_{\text {int }}$ are mainly due to the donor moiety. Also, the charge delocalization and the size of the acceptor modulate the value of $\lambda_{\text {ext }}$ (Table 1 ). This is an interesting feature of fullerene-based DA molecules. As we shall see in further sections, there is much more uncertainty in other quantities that will be the main sources of the error in predicting $k_{C T}$. In general, the reorganization energy, $\lambda$, is a parameter that does not drastically vary by passing from one DFA to another and therefore is not the origin of the error.

\section{Electronic coupling, $V_{D A}$}

The electronic coupling, $V_{D A}$, is known to be sensitive to the mutual orientation of the donor and acceptor sites. In general, many thermally accessible conformations of the D-A complex should be considered. However, we calculated the coupling only for the equilibrium structure of the complexes because all four studied systems are relatively rigid, either due to the presence of only few dihedral angles or due to strong stabilizing $\pi-\pi$ stacking interactions. Thus, we expect that the computed values of $V_{D A}$ (Table 2, and figures SI7-8 in the ESI) are quite reliable. Nevertheless, we are aware that one should account for thermal structural fluctuations if more insight in the values of the $V_{D A}$ is desired.

Note that the CAM-B3LYP, and specially MN15, give slightly smaller values than the rest of DFAs analysed. Interestingly, all DFAs distinguish two clearly different behaviours regarding $V_{D A}^{2}$ : $\mathbf{C}_{60}-\mathrm{ZnTPP}$ and $\mathbf{C}_{60}-\mathrm{TPA}$ on one hand and $\mathbf{C}_{60}-\mathbf{A z}$ and $\mathbf{C}_{59} \mathbf{N}-\mathbf{P c}$ on the other. The latter present $V_{D A}^{2}$ values 3-4 orders of magnitude greater than the former.

Table 2. Electronic coupling squared, $\mathrm{V}_{\mathrm{DA}^{2}}$. All values in $\mathrm{eV}^{2}$.

\begin{tabular}{|c|c|c|c|c|}
\hline DFA & $C_{60}-A z$ & $\mathrm{C}_{60}$-ZnTTP & $\mathrm{C}_{59} \mathrm{~N}-\mathrm{Pc}$ & $\mathrm{C}_{60}$-TPA \\
\hline CAM-B3LYP & $5.40 \cdot 10^{-3}$ & $9.80 \cdot 10^{-6}$ & $2.76 \cdot 10^{-3}$ & $2.13 \cdot 10^{-6}$ \\
\hline MN15 & $6.72 \cdot 10^{-3}$ & $2.19 \cdot 10^{-6}$ & $2.16 \cdot 10^{-4}$ & $4.90 \cdot 10^{-7}$ \\
\hline$\omega B 97 x D$ & $4.97 \cdot 10^{-3}$ & $5.84 \cdot 10^{-5}$ & $5.36 \cdot 10^{-3}$ & $6.10 \cdot 10^{-6}$ \\
\hline M11 & $5.34 \cdot 10^{-4}$ & $2.13 \cdot 10^{-6}$ & $9.99 \cdot 10^{-4}$ & $1.50 \cdot 10^{-5}$ \\
\hline LC- $\omega$ hPBE & $5.24 \cdot 10^{-3}$ & $1.45 \cdot 10^{-5}$ & $3.81 \cdot 10^{-3}$ & $1.51 \cdot 10^{-5}$ \\
\hline LC-TPSS & $6.97 \cdot 10^{-3}$ & $1.83 \cdot 10^{-5}$ & $4.02 \cdot 10^{-3}$ & $2.08 \cdot 10^{-5}$ \\
\hline LC-BLYP & $7.19 \cdot 10^{-3}$ & $1.69 \cdot 10^{-4}$ & $4.06 \cdot 10^{-3}$ & $4.72 \cdot 10^{-5}$ \\
\hline Mean & $5.85 \cdot 10^{-3}$ & $3.92 \cdot 10^{-5}$ & $3.03 \cdot 10^{-3}$ & $1.52 \cdot 10^{-5}$ \\
\hline
\end{tabular}

\section{$\Delta \mathbf{G}^{0}{ }_{\mathrm{CT}}$ calculations}

We have seen already that $\lambda$ has not a critical impact on the evaluation of the $k_{C T}$ for the four systems studied since the variation among them is minor. $\Delta \mathrm{G}^{0}{ }_{\mathrm{CT}}$ is present in the exponential part of Marcus equation as well, thus should be analysed carefully since the errors present in $\Delta \mathrm{G}^{0} \mathrm{CT}$ have an exponential translation in the rate constant. To take place, CT reactions should present negative or slightly positive $\Delta \mathrm{G}^{0}{ }_{\mathrm{CT}}$ values. Computationally, small positive values can be also accepted at DFT level, since other electronic states are usually very close in energy to the ones of interest and therefore, for an accurate (and far more expensive) calculation of these energies one should use multireference methods. Fortunately, for $\mathbf{C}_{60^{-}}$ $\mathbf{A z}, \mathbf{C}_{59} \mathbf{N}-\mathbf{P c}$, and $\mathbf{C}_{60}-\mathrm{TPA}$ experimental $\Delta \mathrm{G}^{0}{ }_{\mathrm{CT}}$ are available and the DFT accuracy is benchmarked against them in Table 3, (and Figures SI9-10 in the ESI). It is usual for a light-harvesting material that the Gibbs energy for the charge separation $\left(\Delta \mathrm{G}^{0} \mathrm{cs}\right)$ is found in the normal Marcus region, therefore $\Delta \mathrm{G}^{\circ}{ }_{\mathrm{CT}}$ 's are expected to be negative in order the charge transfer to occur in a given rate (and it is what happens herein). This is a great difference with respect to the charge recombination processes, which present their $\Delta \mathrm{G}^{0}\left(\Delta \mathrm{G}^{0} \mathrm{CR}\right)$ in the Marcus inverted region. In this manner, the lifetime of the CTS are usually longer. If this is the case, their theoretical evaluation becomes even more challenging. For $\Delta \mathrm{G}^{0} \mathrm{CT}^{\prime} \mathrm{s}$, not as like in the previous variables, it can be seen that the choice of the DFA is crucial for its correct evaluation. LC-whPBE, LC-TPSS, and LC-BLYP always give 
positive $\Delta \mathrm{G}^{0} \mathrm{CT}^{\prime} \mathrm{s}$ when they should be negative. Although $k_{C T}$ can still give reasonable results due to some error cancelation, one expects to get the proper thermodynamics of the cycle depicted in Figure 1. The highest error, $1.57 \mathrm{eV}$, is given by LC-BLYP for $\mathbf{C}_{60}-\mathbf{A z}$. On the other hand, a qualitative good description for $\Delta \mathrm{G}^{0}{ }_{\mathrm{CT}}$ 's is given by MN15, M11, $\omega \mathrm{B} 97 \times \mathrm{D}$ and CAM-B3LYP. For $\mathrm{C}_{60}-\mathrm{ZnTTP}$ system, we expect to have a negative $\Delta \mathrm{G}^{0}{ }_{\mathrm{CT}}$ 's since MN15, CAM-B3LYP, $\omega B 97 X D$, and M11 predict so. It is important to notice that in the exponent of Eq. 6 there is not only $\Delta \mathrm{G}^{0} \mathrm{CT}$, but $\left(\lambda+\Delta \mathrm{G}^{0}{ }_{\mathrm{CT}}\right)^{2}$. It could happen, then, that the correct $k_{C T}$ is reached due to some error compensation effect between incorrect $\Delta \mathrm{G}^{0}{ }_{\text {Ст }}$ and $\lambda$. This, in general, does not occur in our case (see Tables SI1 to SI6 for details). We want to finish this section stressing the fact that $\Delta \mathrm{G}^{0} \mathrm{CT}$ is probably the best tool to discriminate at first sight whether a specific DFA will perform appropriately or not for CT rate constant evaluations, and based on the results of Table 3, MN15, CAM-B3LYP, $\omega B$ 97xD, and M11 are the most promising DFAs. The last three functionals clearly do not predict properly even the qualitative sign of $\Delta \mathrm{G}$.

Table 3. DFT-computed $\Delta \mathrm{G}_{\mathrm{CT}}{ }^{\prime} \mathrm{S}$ and experimental references. All values in $\mathrm{eV}$.

\begin{tabular}{|c|c|c|c|c|}
\hline$\Delta \mathrm{G}^{0}{ }_{\mathrm{CT}}(\mathrm{eV})$ & $C_{60}-A z$ & $\mathrm{C}_{60}-\mathrm{ZnTTP}$ & $\mathrm{C}_{59} \mathrm{~N}-\mathrm{Pc}$ & $\mathrm{C}_{60}-\mathrm{TPA}$ \\
\hline CAM-B3LYP & 0.20 & -0.42 & -0.52 & -0.96 \\
\hline MN15 & -0.02 & -0.73 & -0.76 & -1.41 \\
\hline$\omega B 97 x D$ & 0.35 & -0.09 & 0.03 & -0.23 \\
\hline M11 & 0.47 & -0.15 & -0.08 & -0.04 \\
\hline LC- $\omega$ hPBE & 0.89 & 0.41 & 0.03 & 0.10 \\
\hline LC-TPSS & 1.00 & 0.51 & 0.06 & 0.21 \\
\hline LC-BLYP & 1.30 & 0.80 & 0.12 & 0.96 \\
\hline Experimental & -0.27 & - & -0.24 & -0.31 \\
\hline
\end{tabular}

\section{Charge transfer rate}

After the parameters of the Marcus equation have been examined, we are now in the position to look into the estimated charge transfer rates. We compare the relative errors of calculated values of $\log \left(k_{C T}\right)$ with the experimental data (Table 4, Figure 3 and Figures SI1-SI3 in the ESI). The absolute values are listed in the supporting information (Tables SI1-SI6 in the ESI). The best overall performance is given by the CAM-B3LYP functional, followed by MN15 and $\omega B 97 \times D$ with the mean absolute percentage error (MAPE) error of $6.3 \%, 10.9 \%$, and $14.25 \%$, respectively. The functionals LC-WhPBE, LC-TPSS, and LC-BLYP show worse results due to inaccurate estimates of $\Delta \mathrm{G}^{0}$ Ст. In general, worst functionals always underestimates the rate constants, whereas best functionals tend to slightly overestimate some of them, except for the case of $\mathbf{C}_{60}$-TPA, for which all functionals underestimate $k_{C T}$. The analysis of the DFA errors split by systems clearly shows that CAM-B3LYP, MN15, $\omega B 97 \times D$, and $M 11$ are not only the best functionals in MAPE but they are also the best functionals for each system, as can be seen in Figure 3. It is worth mentioning that all DFAs give very good predictions for $\mathrm{C}_{59} \mathrm{~N}-\mathrm{Pc}$, whilst the biggest error is always found for $\mathrm{C}_{60}-\mathrm{Az}$.

Table 4. Relative errors of the logarithm of the charge transfer rate constant. Mean absolute percentage error (MAPE) for each DFA. Key parameters for the definition of each DFA.

\begin{tabular}{|c|c|c|c|c|c|c|c|}
\hline \multirow[b]{2}{*}{ System } & \multirow[b]{2}{*}{ CAM-B3LYP } & \multicolumn{6}{|c|}{ Relative error $\log \left(k_{c}\right)(\%)$} \\
\hline & & MN15 & $\omega B 97 x D^{[a]}$ & M11 & LC-whPBE & LC-TPSS & LC-BLYP \\
\hline $\mathrm{C}_{60}-\mathrm{Az}$ & 2.42 & 26.72 & -21.61 & -49.12 & -119.83 & -146.33 & -266.88 \\
\hline $\mathrm{C}_{60}-\mathrm{ZnTTP}$ & 7.64 & 0.42 & 5.14 & -7.89 & -55.67 & -75.52 & -121.13 \\
\hline $\mathrm{C}_{59} \mathrm{~N}-\mathrm{Pc}$ & 11.53 & -5.89 & 7.35 & 3.86 & 0.38 & -2.56 & -6.96 \\
\hline $\mathrm{C}_{60}$-TPA & -3.64 & -10.46 & -22.90 & -31.90 & -46.17 & -58.38 & -141.58 \\
\hline MAPE & 6.31 & 10.87 & 14.25 & 23.19 & 55.51 & 70.70 & 134.14 \\
\hline \multicolumn{8}{|l|}{ RSH definition } \\
\hline Full-range $H F_{x}$ coefficient $(\alpha)$ & 0.19 & 0.44 & 0.222036 & 0.428 & 0.00 & 0.00 & 0.00 \\
\hline Long-range $H F_{x}$ coefficient $(\beta)$ & 0.46 & 0.00 & 0.777964 & 0.572 & 1.00 & 1.00 & 1.00 \\
\hline Total $H F_{x}$ at long range $(\alpha+\beta)$ & 0.65 & 0.44 & 1.00 & 1.00 & 1.00 & 1.00 & 1.00 \\
\hline Attenuation parameter $(\omega)$ & 0.33 & - & 0.20 & 0.25 & 0.4 & 0.47 & 0.47 \\
\hline
\end{tabular}

[a] For $\omega$ B97xD functional, $H F x$ and DFAx are treated differently. The full-range DFAx coefficient is 1.0 and the long-range DFAx coefficient is 0 . 


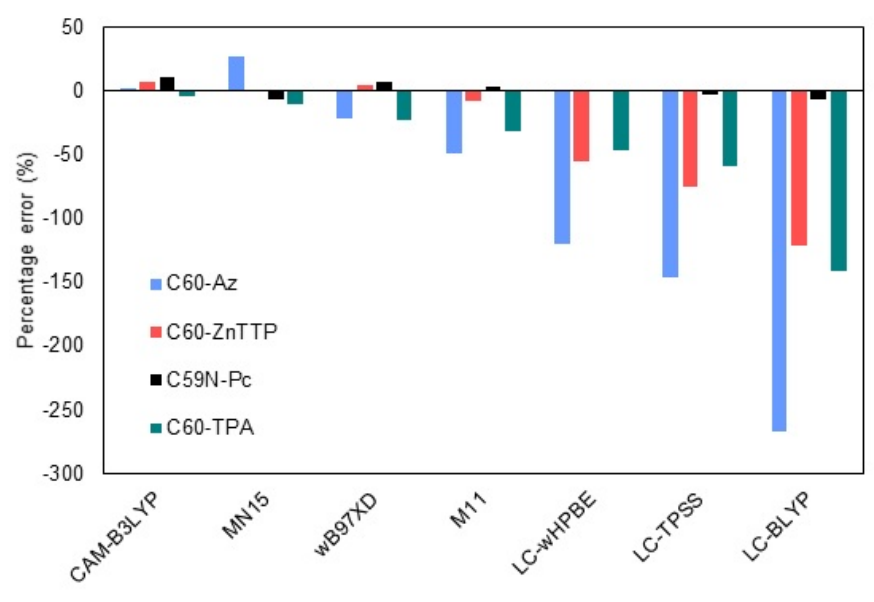

Figure 3. Mean percentage error of the $\log \left(\mathrm{k}_{\mathrm{CT}}\right)$ for the four Donor-Acceptor dyads and the seven DFAs studied in this work.

The DFAs show similar and most accurate values of $\Delta \mathrm{G}^{0} \mathrm{CT}$ and $k_{C T}$ for $\mathrm{C}_{59} \mathrm{~N}$-Pc (Table $\mathrm{SI} 3$ in the ESI). It is worth mentioning that $\mathrm{C}_{60}-\mathrm{Az}$ computed at MN15 level of theory presented two pseudo-degenerate CTS which present almost identical $k_{\mathrm{CT}}$ of $1.13 \cdot 10^{13} \mathrm{~s}^{-1}$ and $1.70 \cdot 10^{13} \mathrm{~s}^{-1}$. We finally reported the CTS leading to the former $k_{C T}$ since the rest of the parameters, and specially the electronic coupling, resembled more to the ones computed with other DFAs, thus indicating that we are targeting the same CTS for all functionals.

The construction of the DFA has a clear impact on the final results. For a general long-range corrected (LC) hybrid scheme, the $1 / r_{12}$ operator for the exchange part of the functional is split in the short-range (SR), and long-range (LR) parts,

$$
\frac{1}{\mathrm{r}_{12}} \equiv \underbrace{\frac{1-\left[\alpha+\beta \cdot \operatorname{erf}\left(\omega \mathrm{r}_{12}\right)\right]}{\mathrm{r}_{12}}}_{\mathrm{SR}}+\underbrace{\frac{\alpha+\beta \cdot \operatorname{erf}\left(\omega \mathrm{r}_{12}\right)}{\mathrm{r}_{12}}}_{\mathrm{LR}}
$$

where $r_{12}$ is the interelectronic distance. On the right-hand side of Eq 10, the first term governs the SR part and the second term governs the LR part of the functional. Note that using this equation, $\alpha$ controls the amount of exact exchange $(\mathrm{HFx})$ added at all interelectronic distances, while $\beta$ governs the extra amount of $\mathrm{HF}_{\mathrm{X}}$ added at LR; and the separation between $\mathrm{SR}$ and $L R$ is controlled by the $\omega$ attenuation parameter. In this context, the exchange-correlation energy, $E_{X C}$, can be expressed as

$$
\begin{gathered}
\mathrm{E}_{\mathrm{XC}}=\alpha \mathrm{E}_{\mathrm{HF}_{\mathrm{X}}, \omega}^{\mathrm{SR}}+(1-\alpha) \mathrm{E}_{\mathrm{DF} A_{\mathrm{X}}, \omega}^{\mathrm{SR}} \\
+(\alpha+\beta) \mathrm{E}_{\mathrm{HF}_{\mathrm{X}}, \omega}^{\mathrm{LR}}+(1-\alpha-\beta) \mathrm{E}_{\mathrm{DFA}_{\mathrm{X}}, \omega}^{\mathrm{LR}}+\mathrm{E}_{\mathrm{C}}
\end{gathered}
$$

where DFA $A_{x}$ corresponds to the (semi)local exchange energy. Eq. 11 can be used for the construction of CAM-B3LYP ( $\alpha=0.19$, $\beta=0.46, \omega=0.33$ Bohr $\left.^{-1}\right)$, MN15 $(\alpha=0.44, \beta=0.00)$, LC $-\omega$ hPBE $\left(\alpha=0.00, \beta=1.00, \omega=0.40\right.$ Bohr $\left.^{-1}\right)$, LC-TPSS $(\alpha=0.00, \beta=1.00$, $\omega=0.47$ Bohr $\left.^{-1}\right)$ and LC-BLYP $\left(\alpha=0.00, \beta=1.00, \omega=0.47\right.$ Bohr $\left.^{-1}\right)$. However, the construction of $\omega B 97 \times D$ follows a slightly different parametrization since it treats differently the $\mathrm{HF}_{\mathrm{X}}$ than the DFAx part, in particular $\omega B 97 \times D$ can be defined as

$$
E_{X C}=c_{x} E_{H F_{X}, \omega}^{S R}+E_{D F A_{X}, \omega}^{S R}+E_{H F_{X}, \omega}^{L R}+E_{C}
$$

where $c_{x}=0.222036$. Similar to theoretical predictions of the UV-visible absorption spectra of fullerenic and non-fullerenic DA molecules, ${ }^{65}$ the amount of exact exchange added to the DFAs through the range-separated scheme appears to be a key parameter to control the performance of DFA for chargetransfer reactions. First, the attenuation parameter should be relatively small (i.e. $<0.4 \mathrm{Bohr}^{-1}$ ) and therefore the transition towards long range region should occur at rather big electronelectron distances to get proper $k_{C T}$ values. One could think that having less $\mathrm{HFx}_{\mathrm{X}}$ at short range region will be somehow compensated by a larger value of the attenuation parameter (to switch to the long-range region at shorter distances, i.e. LCwhPBE, LC-TPSS or LC-BLYP). However, this compensation is not observed and some amount of $\mathrm{HF}_{\mathrm{X}}$ must be added at shortrange to get accurate rates, as happens with CAM-B3LYP (19\%), MN15 (44\%), and $\omega B$ 97xD (22\%). On the other hand, the optimal amount of $\mathrm{HFX}$ at long range needs to be $<100 \%$, as the best DFA have 0.65 (CAM-B3LYP) and 0.44 (MN15) of total HFX at long-range. The same requirement has been reported beneficial, in terms of accuracy of the results, for other longrange properties as electronic excitation energies. ${ }^{66,} 67$ The usage of $(\alpha+\beta)<1$ can be rationalized as a requirement to reproduce the effective dielectric constant of the material $\left(\alpha+\beta=\varepsilon_{\text {eff }}\right)$ that tailors the decay of the XC potential as $\left(\varepsilon_{\text {eff }} r\right)^{-1} .{ }^{66}$, ${ }^{67}$ For M11 functional $\alpha+\beta$ is equal to 1.0. However, it has two other desirable features: (1) the attenuation parameter is relatively low (clearly lower than in LC-BLYP, LC-TPSS or even LCwhPBE), and (2) the $\mathrm{HF}_{\mathrm{X}}$ term at short electron-electron distances is not zero.

\section{Tuning, a look into the future?}

Charge transfer rate constants demand properly taking into account the long-range interactions to be accurately computed, a well-balanced self-interaction error and a proper decay of the exchange-correlation potential. In this and some other fields, range-separated functionals can be tuned to meet such requirements. ${ }^{68}$ Herein we have tested, as a proof of concept, two tuning schemes for LC-BLYP, which is the worst method overall, trying to improve their results for $\mathbf{C}_{60}$-TPA. Our goal was to perform a preliminary study to explore if the tuned-DFAs have the potential to be good candidates to be used for the evaluation of $\log \left(k_{C T}\right)$.

First, we forced LC-BLYP to fulfil Janak's theorem (OT1) by screening the $\omega$ parameter, ${ }^{69}$ as proposed by Kronik and coworkers. ${ }^{70,71}$ The working $\omega$ for OT1 was 0.236 . Similarly, we also tested a novel tuning strategy recently developed by some of us (OT2). ${ }^{72}$ In OT2 the empirically-tuned attenuation parameter is given by a function of a molecular descriptor defined from the linear polarizability and the number of electrons in the molecule. The resulting $\omega$ was determined to be 0.303 . 
Then, charge-transfer rates for OT1 and OT2 were computed (Table 5). It has been reported that the variation of the DFA may change the character of the charge transfer states determined by Equation $2 .{ }^{73}$ We also observe this effect. Since the CT character of the $D^{+}-A^{-}$state increases going from LC-BLYP ( $C S=0.717, C T=0.723)$ to OT1 $(C S=0.930, C T=0.931)$ and OT2 ( $C S=0.982, C T=0.982)$, the $D^{+}-A^{-}$state become almost a pure $C T$ state. The state character for the DFAs can be found in the SI.

Table 5. Marcus equation's parameters and rate constants for LC-BLYP, OT1, and OT2 functionals. All values in eV.

\begin{tabular}{cccccc}
\hline Method & $\boldsymbol{\lambda}_{\text {int }}$ & $\boldsymbol{\lambda}_{\text {ext }}$ & $\boldsymbol{V}_{\boldsymbol{D A}}^{2}$ & $\boldsymbol{\Delta G}$ & $\operatorname{Error} \log \left(\boldsymbol{k}_{\boldsymbol{c}} \boldsymbol{)}\right)$ \\
\hline OT1 & 0.21 & 0.56 & $6.81 \cdot 10-6$ & 0.21 & $-46.2 \%$ \\
OT2 & 0.22 & 0.89 & $9.36 \cdot 10-6$ & -0.15 & $-28.8 \%$ \\
LC-BLYP & 0.26 & 0.53 & $4.72 \cdot 10-5$ & 0.96 & $-141.6 \%$ \\
\hline
\end{tabular}

It is clearly seen that the tuning of range-separated DFAs can have deep positive impact on the accuracy of the obtained results. For the $\mathbf{C}_{60}$-TPA the errors are reduced more than threefold going from pristine LC-BLYP to OT1, and those are divided by more than 1.5 again when moving to OT2. Therefore, a fivefold improvement of the logarithm of the rate constant is achieved using OT2. Interestingly, both OT1 and OT2 tuning schemes give $\omega$ between the values for CAM-B3LYP and $\omega B 97 x D$, which leads to a sharp decrease of the corresponding relative error of computed $k_{\text {CT }}$.

Furthermore, from pristine LC-BLYP to OT2 we are going towards a qualitatively more reliable description of the system since $\Delta \mathrm{G}^{0} \mathrm{CT}$ is becoming closer to the experimental value of $0.31 \mathrm{eV}$. Moreover, OT2 is the only DFA from the LC-BLYP family that presents the proper sign for the $\Delta \mathrm{G}^{0} \mathrm{CT}$ of the $\mathrm{CT}$, which is conceptually very important. Interestingly, the $\lambda_{\text {ext }}$ for OT2 is clearly different from the ones of pristine LC-BLYP and OT1, being closer to the ones for CAM-B3LYP $(0.94 \mathrm{eV})$ and MN15 $(0.97 \mathrm{eV})$ or $\omega \mathrm{B97xD}(0.82 \mathrm{eV})$, which could be an indicator of quality. Nevertheless, more effort should be put into these tuned functionals to be better than other alternatives such as CAM-B3LYP or MN15 for the evaluation of charge transfer processes. In that line, the ideal candidate to be tuned in further work using OT2 procedure is CAM-B3LYP. There are other alternatives to tuned DFAs, such as the new revM11 functional, which is tailored to tackle ground- and excited-state properties. $^{74}$

\section{Conclusions}

We have analysed several factors that can determine the accuracy of the computed charge transfer rate for four fullerene-based systems, namely, $\mathbf{C}_{60}-\mathbf{A z}, \mathbf{C}_{59} \mathbf{N}-\mathbf{P c}, \mathbf{C}_{60}-\mathbf{Z n T P P}$, and $\mathbf{C}_{60}$-TPA, at the DFT level of theory with five well-known range-separated GGA DFAs (CAM-B3LYP, $\omega B$ 97XD, LC- $\omega \mathrm{hPBE}$, LC-TPSS, LC-BLYP) and one meta-hybrid-GGA functional, MN15. We have found that the estimation of the reorganization energy and the electronic coupling given by all benchmarked DFAs have a small impact in the final accuracy of charge transfer rate. On the contrary, $\Delta \mathrm{G}^{0}{ }_{\mathrm{CT}}$ has proven to be a critical parameter for the
CT rate computation. Only the MN15, $\omega B 97 \times D$, and especially CAM-B3LYP functionals are able to describe $\Delta \mathrm{G}^{0}$ CT accurately enough, correctly reproducing their negative sign. These three DFAs give also best results for the $k_{C T}$ estimated using the Marcus theory. Overall, we recommend CAM-B3LYP as the best choice for modelling fullerene-based materials. Tuning the amount of full range and long range $\mathrm{HF}_{\mathrm{X}}$ seems to be a promising strategy that needs to be explored in order to develop more accurate functionals for predicting photophysical properties.

\section{Conflicts of interest}

There are no conflicts to declare.

\section{Acknowledgements}

This work was supported with funds from the Ministerio de Economía y Competitividad (MINECO) of Spain (project CTQ201785341-P to M.S. and A.A.V.), the Spanish government MICINN (project PGC2018-098212-B-C22 to J.M.L), and the Generalitat de Catalunya (project 2017SGR39 to M.S. and J.M.L.). We thank the Spanish government for the predoctoral grant to P.B.-S. (FPU17/02058). We are also grateful for the computational time financed by the Consorci de Serveis Universitaris de Catalunya (CSUC).

\section{Notes and references}

1. United Nations Home Page, https://www.un.org/sustainabledevelopment/, (accessed 20/9/2020, 2020).

2. N. Armaroli and V. Balzani, Angew. Chem. Int. Ed., 2007, 46, 52.

3. Y. He and Y. Li, Phys. Chem. Chem. Phys., 2011, 13, 1970.

4. D. Jariwala, V. K. Sangwan, L. J. Lauhon, T. J. Marks and M. C. Hersam, Chem. Soc. Rev., 2013, 42, 2824.

5. G. Chen, J. Seo, C. Yang and P. N. Prasad, Chem. Soc. Rev., 2013, 42, 8304.

6. S. Günes, H. Neugebauer and N. S. Sariciftci, Chem. Rev., 2007, 107, 1324.

7. J. L. Delgado, P.-A. Bouit, S. Filippone, M. Á. Herranz and N. Martín, Chem. Commun., 2010, 46, 4853.

8. M. Rudolf, S. V. Kirner and D. M. Guldi, Chem. Soc. Rev., 2016, 45, 612.

9. Y. Huang, E. J. Kramer, A. J. Heeger and G. C. Bazan, Chem. Rev., 2014, 114, 7006.

10. J. L. Segura, N. Martín and D. M. Guldi, Chem. Soc. Rev., 2005, 34, 31.

11. A. F. Voter, in Radiation Effects in Solids, eds. K. E. Sickafus and E. A. Kotomin, NATO Publishing Unit, Dordretch, The Netherlands, 2007, pp. 1.

12. A. J. Stasyuk, O. A. Stasyuk, S. Filippone, N. Martin, M. Solà and A. A. Voityuk, Chem. Eur. J., 2018, 24, 13020.

13. A. J. Stasyuk, O. A. Stasyuk, M. Solà and A. A. Voityuk, Chem. Eur. J., 2020, 26, 10896.

14. A. J. Stasyuk, O. A. Stasyuk, M. Solà and A. A. Voityuk, Chem. Commun., 2019, 55, 11195. 

Voityuk, S. Cuesta, M. Solà, D. M. Guldi and N. Martín, Angew. Chem. Int. Ed., 2019, 58, 6932.

16. M. Dierksen and S. Grimme, J. Phys. Chem. A, 2004, 108, 10225. M. J. G. Peach, P. Benfield, T. Helgaker and D. J. Tozer, J. Chem. Phys., 2008, 128, 044118.

18. M. R. Silva-Junior, M. Schreiber, S. P. A. Sauer and W. Thiel, J. Chem. Phys., 2008, 129, 104103.

19. M. Isegawa, R. Peverati and D. G. Truhlar, J. Chem. Phys., 2012, 137, 244104.

20. A. D. Laurent and D. Jacquemin, Int. J. Quantum Chem, 2013, 113, 2019.

21.

C. Suellen, R. G. Freitas, P.-F. Loos and D. Jacquemin, J. Chem. Theory Comput., 2019, 15, 4581. R. Grotjahn and M. Kaupp, J. Chem. Theory Comput., 2020, 16, 5821.

A. Ottochian, C. Morgillo, I. Ciofini, M. J. Frisch, G. Scalmani and C. Adamo, J. Comput. Chem., 2020, 41, 1242. A. Dreuw and M. Head-Gordon, Chem. Rev., 2005, 105, 4009. Y. Tawada, T. Tsuneda, S. Yanagisawa, T. Yanai and K. Hirao, J. Chem. Phys., 2004, 120, 8425.

26. E. Livshits and R. Baer, Phys. Chem. Chem. Phys., 2007, 9, 2932.

M. E. Casida and M. Huix-Rotllant, Annu. Rev. Phys. Chem., 2012, 63, 287. C. Adamo and D. Jacquemin, Chem. Soc. Rev., 2013, 42, 845. F. Santoro and D. Jacquemin, Wiley Interdiscip. Rev. Comput. Mol. Sci., 2016, 6, 460. A. D. Becke, J. Chem. Phys., 1993, 98, 5648.

C. Lee, W. Yang and R. G. Parr, Phys. Rev. B, 1988, 37, 785. R. Krishnan, J. S. Binkley, R. Seeger and J. A. Pople, J. Chem. Phys., 1980, 72, 650.

S. Grimme, J. Antony, S. Ehrlich and H. Krieg, J. Chem. Phys., 2010, 132, 154104.

S. Grimme, S. Ehrlich and L. Goerigk, J. Comput. Chem. 2011, 32, 1456.

A. Troisi, Faraday Discuss., 2013, 163, 377. Chem. Acc., 2015, 134, 12.

A. J. Stasyuk, O. A. Stasyuk, M. Solà and A. A. Voityuk, Chem. Commun., 2020, 56, 352.

F. Weigend and R. Ahlrichs, Phys. Chem. Chem. Phys., 2005, 7, 3297.

M. J. Frisch, G. W. Trucks, H. B. Schlegel, G. E. Scuseria, M. A. Robb, J. R. Cheeseman, G. Scalmani, V. Barone, G. A. Petersson, H. Nakatsuji, X. Li, M. Caricato, A. V. Marenich, J. Bloino, B. G. Janesko, R. Gomperts, B. Mennucci, H. P. Hratchian, J. V. Ortiz, A. F. Izmaylov, J. L. Sonnenberg, Williams, F. Ding, F. Lipparini, F. Egidi, J. Goings, B. Peng, A. Petrone, T. Henderson, D. Ranasinghe, V. G. Zakrzewski, J. Gao, N. Rega, G. Zheng, W. Liang, M. Hada, M. Ehara, K. Toyota, R. Fukuda, J. Hasegawa, M. Ishida, T. Nakajima, Y. Honda, O. Kitao, H. Nakai, T. Vreven, K. Throssell, J. A. Montgomery Jr., J. E. Peralta, F. Ogliaro, M. J. Bearpark, J. J. Heyd, E. N. Brothers, K. N. Kudin, V. N. Staroverov, T. A. Keith, R. Kobayashi, J. Normand, K. Raghavachari, A. P. Rendell, J. C. Burant, S. S. Iyengar, J. Tomasi, M. Cossi, J. M. Millam, M. Klene, C. Adamo, R. Cammi, J. W. Ochterski, R.

L. Martin, K. Morokuma, O. Farkas, J. B. Foresman and D. J. Fox, Gaussian 16 Rev. C.01, Wallingford, CT, 2016.

40. A. V. Luzanov and O. A. Zhikol, Int. J. Quantum Chem., 2010, 110, 902.

41. F. Plasser and H. Lischka, J. Chem. Theory Comput., 2012, 8, 2777.

42. A. Klamt and G. Schüürmann, J. Chem. Soc., Perkin Trans. 2, 1993, 799.

43. J. Tomasi, B. Mennucci and R. Cammi, Chem. Rev., 2005, 105, 2999.

44. J. L. Pascual-Ahuir, E. Silla and I. Tuñon, J. Comput. Chem., 1994, 15, 1127.

45. A. Klamt, J. Phys. Chem., 1996, 100, 3349.

46. R. A. Marcus, Rev. Mod. Phys., 1993, 65, 599.

47. P. F. Barbara, T. J. Meyer and M. A. Ratner, J. Phys. Chem., 1996, 100, 13148.

48. R. A. Marcus, J. Phys. Chem., 1994, 98, 7170.

49. R. J. Cave and M. D. Newton, Chem. Phys. Lett., 1996, 249, 15.

50. A. A. Voityuk and N. Rösch, J. Chem. Phys., 2002, 117, 5607.

51. Q. Wu and T. Van Voorhis, Phys. Rev. A, 2005, 72, 024502.

52. A. A. Voityuk, J. Phys. Chem. C, 2013, 117, 2670.

53. H. S. Yu, X. He, S. L. Li and D. G. Truhlar, Chem. Sci., 2016, 7, 5032.

54. R. Peverati and D. G. Truhlar, J. Phys. Chem. Lett., 2011, 2, 2810.

55. T. Yanai, D. P. Tew and N. C. Handy, Chem. Phys. Lett., 2004, 393, 51.

56. J.-D. Chai and M. Head-Gordon, Phys. Chem. Chem. Phys., 2008, 10, 6615.

57. O. A. Vydrov and G. E. Scuseria, J. Chem. Phys., 2006, 125, 234109.

58. T. M. Henderson, A. F. Izmaylov, G. Scalmani and G. E. Scuseria, J. Chem. Phys., 2009, 131, 044108.

59. J. Tao, J. P. Perdew, V. N. Staroverov and G. E. Scuseria, Phys. Rev. Lett., 2003, 91, 146401.

60. H. likura, T. Tsuneda, T. Yanai and K. Hirao, J. Chem. Phys., 2001, 115, 3540.

61. T. Makinoshima, M. Fujitsuka, M. Sasaki, Y. Araki, O. Ito, S. Ito and N. Morita, J. Phys. Chem. A, 2004, 108, 368.

62. G. Rotas, J. Ranta, A. Efimov, M. Niemi, H. Lemmetyinen, N. Tkachenko and N. Tagmatarchis, ChemPhysChem, 2012, 13, 1246.

63. D. M. Guldi, C. Luo, M. Prato, E. Dietel and A. Hirsch, Chem. Comm., 2000, 373.

64. J. P. Martínez, M. Solà and A. A. Voityuk, J. Comput. Chem., 2016, 37, 1396.

65. Y. Ren, M.-Y. Li, Y.-X. Song, M.-Y. Sui, G.-Y. Sun, X.-C. Qu, P. Xie and J.-L. Lu, J. Photochem. Photobiol. A, 2021, 407, 113087.

66. P. Kimber and F. Plasser, Phys. Chem. Chem. Phys., 2020, 22, 6058.

67. A. K. Manna, S. Refaely-Abramson, A. M. Reilly, A. Tkatchenko, J. B. Neaton and L. Kronik, J. Chem. Theory Comput., 2018, 14, 2919.

68. T. Körzdörfer and J.-L. Brédas, Acc. Chem. Res., 2014, 47, 3284.

69. J. F. Janak, Phys. Rev. B, 1978, 18, 7165.

70. N. Kuritz, T. Stein, R. Baer and L. Kronik, J. Chem. Theory Comput., 2011, 7, 2408.

71. T. Stein, L. Kronik and R. Baer, J. Am. Chem. Soc., 2009, 131, 2818. 
72. P. Besalú-Sala, S. P. Sitkiewicz, P. Salvador, E. Matito and J. M. Luis, Phys. Chem. Chem. Phys., 2020, 22, 11871.

73. S. A. Mewes, F. Plasser and A. Dreuw, J. Chem. Phys., 2015, 143, 171101.

74. P. Verma, Y. Wang, S. Ghosh, X. He and D. G. Truhlar, J. Phys. Chem. A, 2019, 123, 2966. 


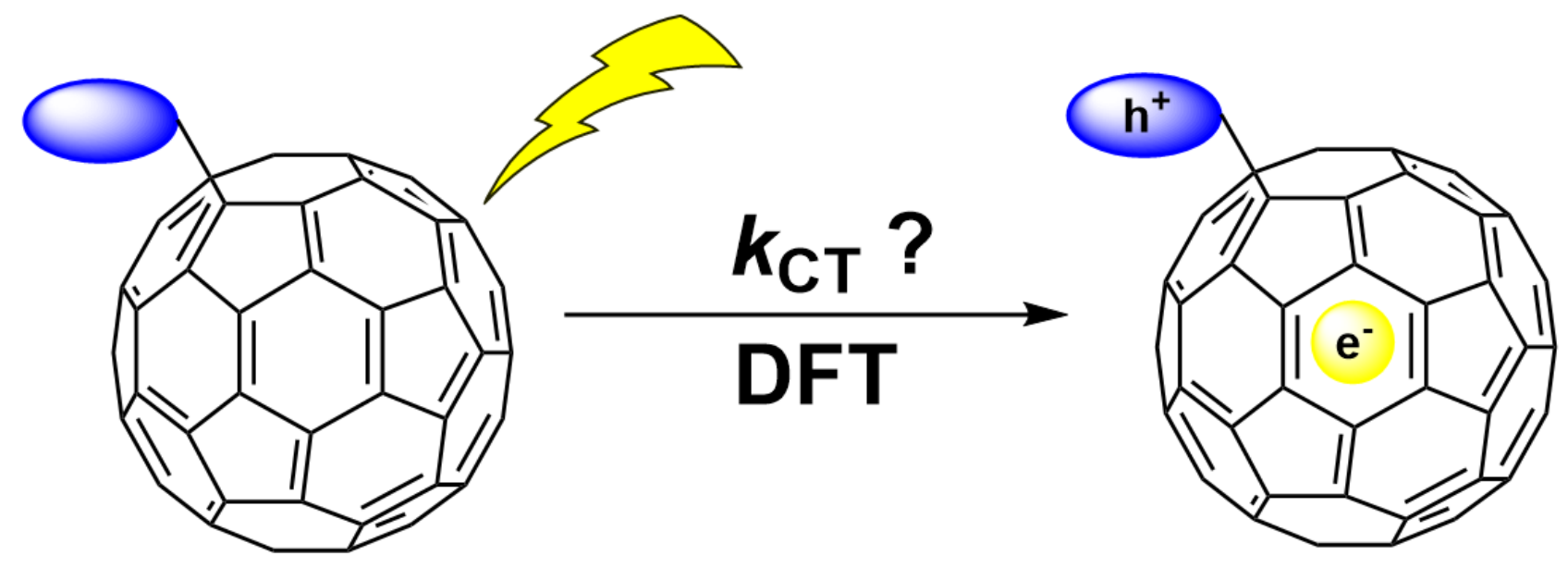

An accurate description of the charge-transfer rate constants $\left(k_{\mathrm{CT}}\right)$ is required for the rational design of fullerene-based molecular heterojunction dye-sensitized solar cells. We have assessed the performance of five long-range corrected hybrid functionals and a meta-GGA functional, putting attention on all the parameters that are used for the calculation of $k_{\mathrm{CT}}$ within the Marcus equation, as well as the impact of the different range-separation schemes on the final results. 